EPJ Web of Conferences 41, 02038 (2013)

DOI: $10.1051 /$ epjconf/20134102038

(C) Owned by the authors, published by EDP Sciences, 2013

\title{
Investigations of ultrafast dynamics in electronically excited alkylbenzenes
}

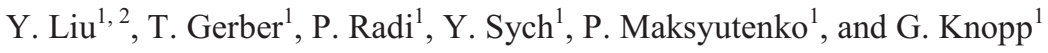 \\ ${ }^{1}$ Paul Scherrer Institute, Villigen 5232, Switzerland \\ ${ }^{2}$ Wuhan Institute of Physics and Mathematics, Chinese Academy of Sciences, Wuhan 430071, China
}

\begin{abstract}
We investigate ultrafast dynamics in electronically excited states of some typical alkylbenzenes by time-resolved two-colour four wave mixing and velocity map imaging as complementary methods. In this context an upgraded double-sided timeresolved velocity map imaging setup is also proposed.
\end{abstract}

\section{Introduction}

Ultrafast electronic relaxation processes play a central role in photochemistry. As the prototypical aromatic species benzene molecules have been the subject of a great number of investigations. Compared with benzene, the non-degeneracy of states in alkyl-benzenes leads to a higher density of the vibrational levels and to a reduction of symmetry forbidden interactions [1]. Hence, higher coupling rates for non-adiabatic transitions are expected.

The absorption of alkyl-substituted benzenes between 190-270 nm is assigned to the excitation of the phenyl ring [2]. The corresponding excited states are stable with respect to dissociation that consequently occurs indirectly after conversion to lower electronic states. The photo excitation of the $\mathrm{S}_{1}$ state of e.g. ethylbenzene subsequently results in different relaxation pathways with distinct time constants and different translational energies of the photo fragments [3]. At wavelength below 266 $\mathrm{nm}$ the lifetime of the $\mathrm{S}_{1}$ state is determined by internal conversion (IC) $\mathrm{S}_{1} \rightarrow \mathrm{S}_{0}$ and by intersystem crossing (ISC) $\mathrm{S}_{1} \rightarrow \mathrm{T}_{1}$. The $\mathrm{T}_{1}$ state decay can occur either barrierless via $\mathrm{T}_{1} \rightarrow \mathrm{S}_{0}$ ISC or by direct dissociation over a barrier. The latter process is expected to dominate the $\mathrm{T}_{1}$ relaxation at $248 \mathrm{~nm}$. We apply time-resolved two-colour four wave mixing spectroscopy (TCFWM) [4] and time-resolved photoelectron imaging (TRPEI) [5] to monitor the electronic relaxation of some typical alkylbenzenes in real time. For further improvement an upgraded double-sided time-resolved velocity map imaging (VMI) setup for enhanced investigation of the complicated dissociation dynamics is proposed.

\section{Dispersed time-resolved TCFWM}

Dispersed time-resolved TCFWM spectroscopy has been used to investigate excited states of alkylbenzenes, including ethylbenzene and propylbenzene. Time-resolved FWM methods implicate the coherent interaction of three light pulses with a medium to generate a signal pulse. Two pump pulses at $265 \mathrm{~nm}(\sim 37735 \mathrm{~cm}-1)$ simultaneously cross the sample, interfere, and generate a polarization that comprises information about the excited state dynamics. The delayed probe pulse is set to $\sim 790 \mathrm{~nm}$ in resonance with $\mathrm{S}_{1} \rightarrow \mathrm{S}_{2}$ transitions as depicted in Fig.1 (a). The bimodal spectral distribution $(\Delta \sim 27$

This is an Open Access article distributed under the terms of the Creative Commons Attribution License 2.0, which permits unrestricted use, distribution, and reproduction in any medium, provided the original work is properly cited. 
cm-1) of the dispersed FWM signal (Fig.1 (b)) may be due to ethyl group torsions close to the origin of the $S_{1}$ state. Low energy vibrations in $S_{1}$, with a separation of $29 \mathrm{~cm}-1$, have been verified in REMPI experiments in Ref. [6]. Apart from that, the measured FWM signals exhibit a bi-exponential dependence upon time delay for all spectral components within the bandwidth of the laser pulses. An ultrafast decay with a time constant of $\tau_{1} \sim 1.4 \mathrm{ps}$ and a second one with $\tau_{2}>20 \mathrm{ps}$ have been derived by a least-square data fit. The fast decline can be interpreted as being caused by vibrational energy redistribution and Frank-Condon mismatch, while the second one rather reflects the population decay in $S_{1}$. Though $\tau_{2}$ tends to slightly rise with increasing detection wavelength, no significant correlation between relaxation time and spectral position could be unambiguously asserted, incorporating the error of the fitting procedure. More clarifying details on the dissociation dynamics can be expected from time-resolved VMI experiments, measuring the energy and angular distributions of the produced photofragments and electrons.

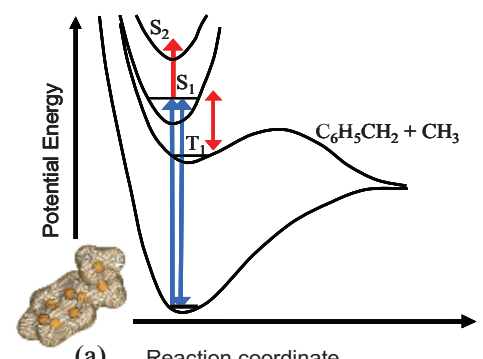

(a) Reaction coordinate

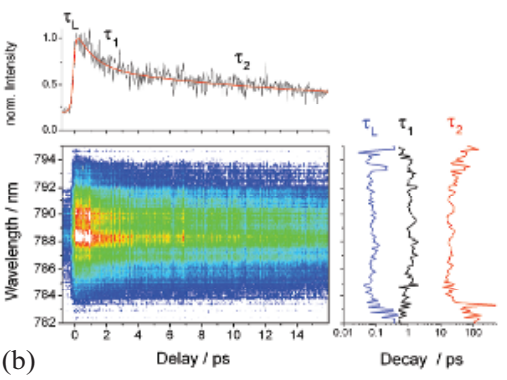

(b)

Fig. 1. Excitation scheme (a) and experimental and fitting results (b) of dispersed time-resolved TCFWM on ethylbenzene.

\section{A double-sided time-resolved VMI setup}

In earlier experiments we have applied TRPEI to investigate o-xylene [7]. The angular distributions of the ejected electrons and their kinetic energy can be obtained from the photoelectron images. Photoelectron images have been detected after a $400 \mathrm{~nm}$ two-photon absorption to the o-xylene $\mathrm{S}_{2}$ state (origin at $\sim 45400 \mathrm{~cm}^{-1}$ ). Fig. 2 (a) depicts the angular distribution of the three measured electron bands. The $1^{\text {st }}$ band relates to the $S_{1}$ state while the others reflect the population in $S_{2}$. The rapid decay of the $2^{\text {nd }}$ and $3^{\text {rd }}$ bands with increasing delay time (Fig. 2 (b)), reveals that population in $\mathrm{S}_{2}$ undergoes an ultrafast IC into vibrationally excited lower states. The relaxation time due to the IC process has been determined to be $\sim 60 \mathrm{fs}$.

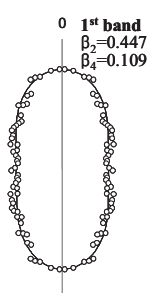

(a) 180

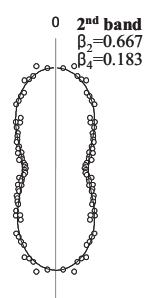

180

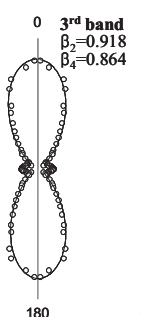

(b)

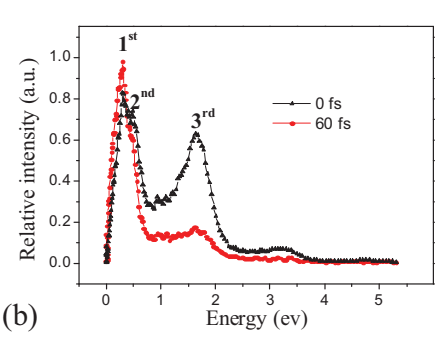

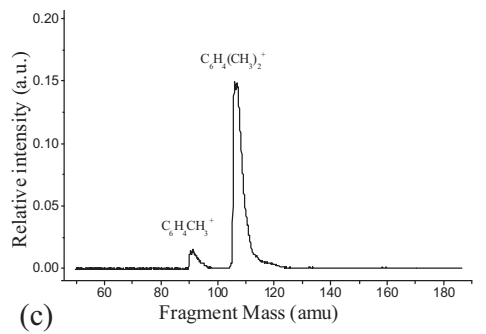

(c)

Fig. 2. (a) Angular distributions; (b) Kinetic energy distributions; (c) A typical TOF mass spectrum.

However, most of the studies by TRPEI are limited by the assumption that no dissociation occurs during the pump-probe processes or that interference from fragments can be neglected and additionally, in conventional VMI experiments the time of flight (TOF) mass resolution is very low, 
as e.g. is shown in Fig 2 (c). The given limitations confine a more detailed investigation of alkylbenzenes. To overcome these restrictions, we assemble a double-sided time-resolved VMI setup, simultaneously collecting energy and momentum information about electrons and coincident ions, which cannot be achieved by photoelectron imaging alone [8]. Moreover, we intend to preserve the longitudinal focus condition for TOF mass resolution on our setup while maintaining the lateral focus for optimal VMI energy resolution simultaneously, which can be achieved by the implementation of additional, especially optimized, electrical lenses. A sketch of the new design is shown in Fig. 3.

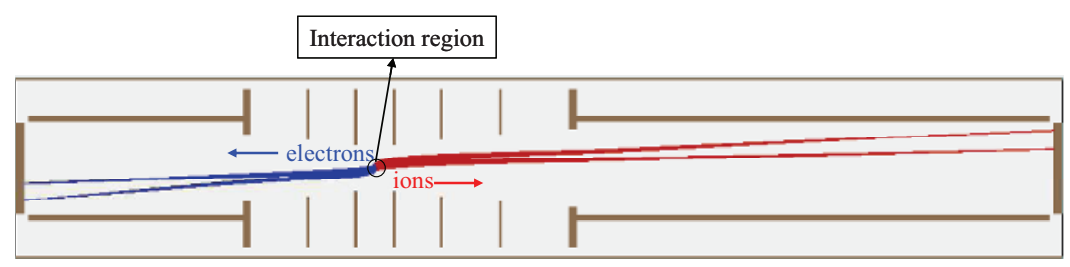

Fig. 3. A sketch of the upgraded double-sided time-resolved VMI setup.

By simulations (Simion 8.1) we can demonstrate that it is possible to achieve velocity-resolving capabilities of $<1 \%$ (full range regime) in conjunction with an optimal TOF mass resolution. Fig. 4 compares the mass resolving capability of our new design (a) with a conventional VMI setup (b) [7]. The resolving power of the new design exceeds the conventional one by almost ten times.
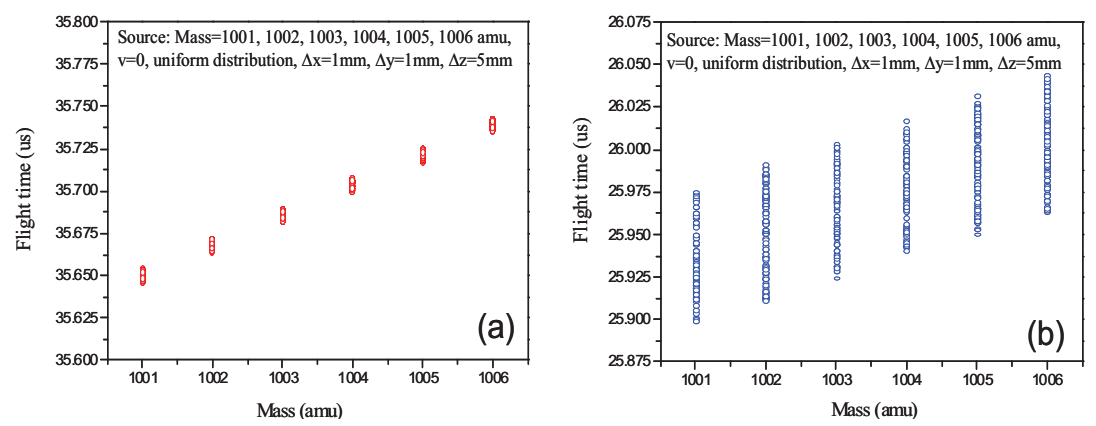

Fig. 4. TOF focus profile of ions with $\mathrm{m} / \mathrm{q}=1001-1006$ for new design (a) and a conventional setup (b).

\section{References}

1. P. Farmanara, V. Stert, W. Radloff, I. V. Hertel, J. Phys. Chem. A 105, 5613 (2001).

2. C. K. Ni, Y. T. Lee, Int. Reviews in Physical Chemistry 23, 187 (2004).

3. C. L. Huang, J. C. Jiang, Y. T. Lee, C. K. Ni, J. Chem. Phys. 115, 7034 (2002).

4. A. M. Walser, M. Meisinger, P. P. Radi, T. Gerber, G. Knopp, Phys. Chem. Chem. Phys. 11, 8456 (2009).

5. T. Suzuki, Annu. Rev. Phys. Chem. 57, 555 (2006).

6. F. Gunzer, J. Grotemeyer, Phys. Chem. Chem. Phys. 4, 5966 (2002).

7. Y. Liu, B. Tang, H. Shen, S. Zhang, B. Zhang, Opt. Express 18, 5791 (2010).

8. C. S. Lehmann, N. B. Ram, D. Irimia, M. H. M. Janssen, Faraday Discuss. 153, 173 (2011). 